\title{
Adequacy of control of cardiovascular risk factors in ambulatory patients with type 2 diabetes attending diabetes out-patients clinic at a county hospital, Kenya
}

Mercy W. Kimando ${ }^{1}$, Frederick C. F. Otieno ${ }^{1 *} \mathbb{D}$, Elijah N. Ogola ${ }^{1}$ and Kenn Mutai ${ }^{2}$

\begin{abstract}
Background: Type 2 diabetes is associated with substantial cardiovascular morbidity and mortality arising from the high prevalence of cardiovascular risk factors such as hypertension, dyslipidaemia, obesity, poor glycaemic control and albuminuria. Adequacy of control of these risk factors determines the frequency and outcome of cardiovascular events in the patients. Current clinical practice guidelines emphasize primary prevention of cardiovascular disease in type 2 diabetes. There is scarce data from the developing countries, Kenya included, on clinical care of patients with type 2 diabetes in the regions that are far away from tertiary health facilities. So we determined the adequacy of control of the modifiable risk factors: glycaemic control, hypertension, dyslipidemia, obesity and albuminuria in the study patients from rural and peri-urban dwelling.
\end{abstract}

Methods: This was a cross-sectional study on 385 randomly selected ambulatory patients with type 2 diabetes without overt complications. They were on follow up for at least 6 months at the Out-patient diabetes clinic of Nyeri County Hospital, a public health facility located in the central region of Kenya.

Results: Females were $65.5 \%$. The study subjects had a mean duration of diabetes of 9.4 years, IQR of 3.0-14 years. Their mean age was 63.3 years, IQR of 56-71 years.

Only $20.3 \%$ of our subjects had simultaneous optimal control of the three (3) main cardiovascular risk factors of hypertension, high LDL-C and hyperglycaemia at the time of the study. The prevalence of cardiovascular risk factors were as follows: HbA1c above $7 \%$ was $60.5 \%$ (95\% Cl, 55.6-65.5), hypertension, $49.6 \%$ of whom $76.6 \%(95 \% \mathrm{Cl}, 72.5-80.8)$ were poorly controlled. High LDL-Cholesterol above $2.0 \mathrm{mmol} / \mathrm{L}$ was found in $77.1 \%$ (95\% Cl 73.0-81.3) and Albuminuria occurred in 32.7\% (95\% Cl 27.8-37.4). The prevalence of the other habits with cardiovascular disease risk were: excess alcohol intake at 26.5\% (95\% Cl 27.8-37.4) and cigarette-smoking at 23.6\%. A modest $23.4 \%$ of the treated patients with hypertension attained target blood pressure of $<140 / 90 \mathrm{mmHg}$. Out of a paltry $12.5 \%$ of the statin-treated patients and others not actively treated, only $22.9 \%$ had LDL-Cholesterol of target $<2.0 \mathrm{mmol} / \mathrm{L}$.

There were no obvious socio-demographic and clinical determinants of poor glycaemic control. However, old age above 50 yrs., longer duration with diabetes above 5 yrs. and advanced stages of CKD were significantly associated with hypertension. Female gender and age, statin non-use and socio-economic factor of employment were the significant determinants of high levels of serum LDL-cholesterol.

(Continued on next page)

\footnotetext{
* Correspondence: cfotieno@gmail.com

'Department of Clinical Medicine and Therapeutics, College of Health

Sciences, University of Nairobi, Nairobi, Kenya

Full list of author information is available at the end of the article
} 
(Continued from previous page)

Conclusion: The majority of the study patients attending this government-funded health facility had high prevalence of cardiovascular risk factors that were inadequately controlled. Therefore patients with type 2 diabetes should be riskstratified by their age, duration of diabetes and cardiovascular risk factor loading. Consequently, composite risk factor reduction strategies are needed in management of these patients to achieve the desired targets safely. This would be achieved through innovative care systems and modes of delivery which would translate into maximum benefit of primary cardiovascular disease prevention in those at high risk. It is a desirable quality objective to have a higher proportion of the patients who access care benefiting maximally more than the numbers we are achieving now.

\section{Background}

The prevalence of cardiovascular disease is strikingly increased in persons with diabetes more than those without diabetes [1]. Cardiovascular events make about eighty $(80 \%)$ percent of the morbidity and mortality in the patients with type 2 diabetes [2].

Type 2 diabetes mellitus is often co-morbid with the cardiovascular risk conditions that include: modifiable ones, being hypertension, dyslipidemia, obesity, smoking and poor glycemic control, and the non-modifiable ones of aging and genes that have been associated with enhanced cardiovascular morbidity. The developed world has experienced improved care and outcomes in patients with type 2 diabetes but quite a high proportion of treated patients have not achieved desired targets in glucose, blood pressure and cholesterol control [3].

Sub-Saharan Africa, like the rest of the world, is experiencing an increasing prevalence of diabetes alongside other non-communicable diseases. The prevalence of diabetes ranges from.

4.3\% in sub-Saharan Africa, $6.7 \%$ in Europe, $10.5 \%$ in North America and the Caribbean to $10.9 \%$ in the Middle East and North Africa [4]. These numbers project the care demands of people with type 2 diabetes now and in the future that sub-Saharan Africa may not be adequately prepared for.

The INTERHEART study, which included participants from sub-Saharan Africa, identified nine cardiovascular risk factors, (diabetes, hypertension, dyslipidaemia, smoking, obesity, unhealthy diet, physical inactivity, alcohol consumption, psychosocial stresses), that explained more than $90 \%$ of the coronary events in the study [5]. That the prevalence of cardiovascular risk factors in type 2 diabetes is high, and the consequences of clinical events is burdensome to patients, their families, and society cannot be overemphasized.

Factors that affect optimal control of these risk factors include access to care, cost of medication and care, socio- economic factors at national and individual levels and psychosocial support system [6]. Sustained adequate cardiovascular risk factor control in a high proportion of patients with type 2 diabetes remains elusive. Therefore more studies are needed, especially in resource- constrained settings, to evaluate the care provision for Quality Improvement, to determine the proportions of patients not attaining targets and underlying reasons to intervene on. This study was conducted to audit the care provided to patients with type 2 diabetes in the public health facility.

\section{Methods \\ Study design and population}

This was a cross-sectional study conducted over 4month period between December 2014 and March 2015 at the diabetes out-patient clinic in Nyeri level 5 hospital, a public health facility. About 7000 patients with diabetes (both types 1 and 2, old and new) were seen in the previous calender year. This was an audit of the clinical care delivered to the patients with type 2 diabetes at this health facility.

This clinic is held once-weekly on Fridays except on public holidays. It has dedicated staff of a Medical Officer, Nurses and an Educator who is a dietitian, but not specifically trained in diabetes care. Their activities at the clinic include weight and height measurements, Blood Pressure determination, and random blood glucose (after the patient has paid for this test). There are only a very small number of patients who perform self-monitoring of blood glucose. Diabetes Education for Self-Management is offered to the clinic attendees as a group, individual approach is only an occasional encounter.

The participants targeted were patients with file diagnosis of type 2 diabetes, previously diagnosed the standard way by the primary physician. The patients were randomly selected from amongst the clinic attendees of the day. Those included in the study were aged 30 years and above, on follow-up for at least 6 months, on either oral anti-diabetic medication alone or in combination with insulin or diet-only. The flow chart, Fig. 1, below depicts the enrolment process.

\section{Ethics, consent and approval}

The study was approved by the Depart of Clinical Medicine and Therapeutics and the Ethics Review Committees of UoN/KNH and of the Nyeri County 


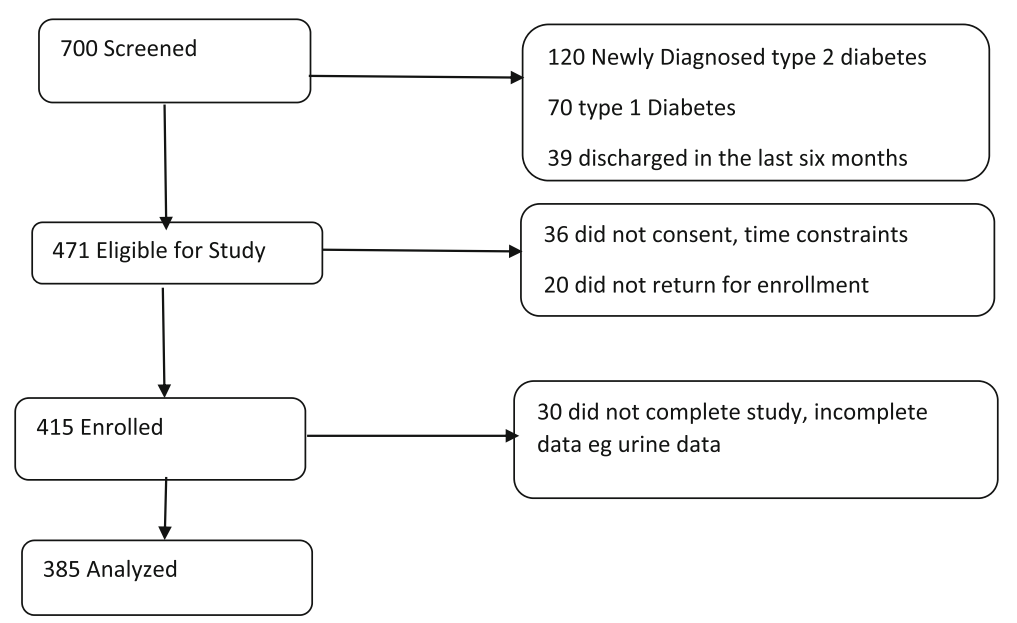

Fig. 1 A flow chart of subject recruitment, enrolment into and activities in the study

hospital. Full explanation was given to the eligible patient and informed written consent was obtained from each subject before enrolment (Fig. 2).

\section{Study assessments and clinical methods}

A complete history was taken from each study participant for socio-demographic information, relevant clinical information on the diabetes mellitus including its treatment and any hypertension or cardiovascular disease. Social habits of alcohol intake and cigarette smoking were documented. The age was verified with patient's national identity card. The marital status was recorded as reported by the study patient. The last prescription and/or the file notes of the last review was used to corroborate the treatment information. Full clinical examination was performed.

Blood pressure was measured by the doctor after the study participant had rested for about 10-min from the time of arrival. While seated with the arm in a comfortable position at the level of the heart, systolic blood pressure was taken at the 1st Korotkoff sound and the diastolic blood pressure taken at the disappearance of Korotkoff sound on a manual mercury sphygmomanometer, both values were measured to the nearest $\mathrm{mmHg}$. The presence of hypertension was taken at BP $\geq 140 / 90 \mathrm{mmHg}$, as classified per JNC 8 [7]. Waist and Hip circumferences were measured in centimeters on the transverse plane at the level of the narrowest part of torso between the lowest rib and the top of pelvis as seen on the anterior view at the end of normal expiration for waist circumference. This was at the level of greater trochanter on the transverse plane for hip circumference. Waist hip ratio (WHR) was calculated as a ratio of Waist Circumference to Hip Circumference [8] and classified as per the NCEP/ATP III guidelines respectively [9]. Height was measured on the patient standing without shoes, the back against the wall on tape, to the nearest ten centimeter. Weight was taken on

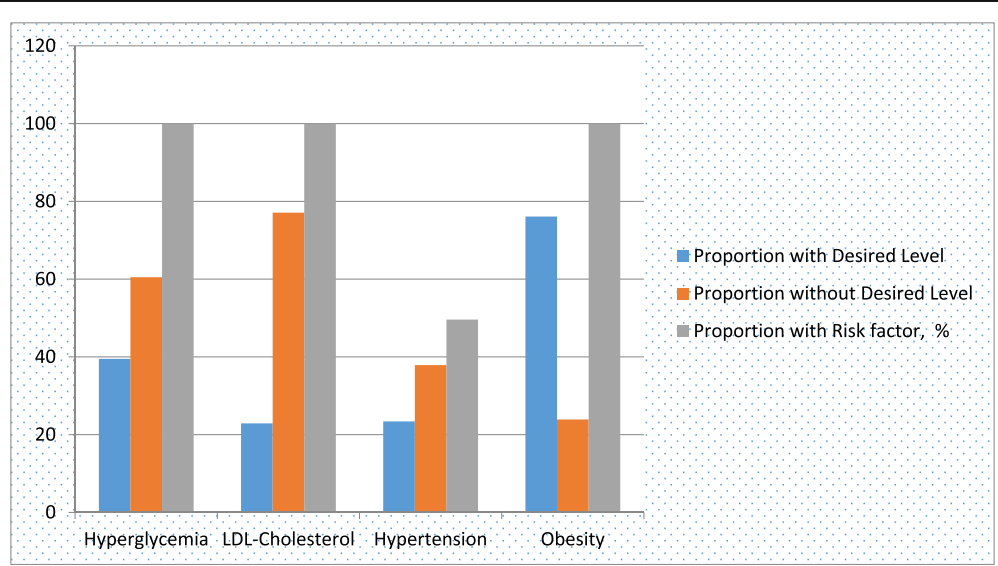

Fig. 2 Bar Chart depicting selected Cardiovascular Risk Factors in control and the number at risk amongst the study subjects 
an electronic weighing machine to the nearest $0.5 \mathrm{~kg}$. Body mass index (BMI) was calculated as weight in kilograms divided by height in meters squared and classified [10].

\section{Laboratory methods}

The patients were advised to come to the diabetes clinic day, every Friday morning fasted. An 8 ml- venous blood sample was asceptically drawn from the cubital fossa. A six (6) $\mathrm{ml}$-sample was collected in clot-activated vacutainers for serum lipid profile and the remaining $2 \mathrm{ml}$-sample into EDTA vacutainer for HbA1c determination. The samples were stored in cool boxes with dry ice carbon dioxide at 2 to $8{ }^{\circ} \mathrm{C}$, then delivered to the laboratory of the University's Department of Clinical medicine for assays later. HBA1c was processed by glycohemoglobin ion exchange resin method from ERBA MANNHEIM Gmbh at the laboratory. HbA1c $>7.0 \%$ was considered sub-optimal control.

Lipid profile was analyzed using Human Gmbh kit. Total cholesterol was measured using the CHOD-PAP method based on Trinders Methodology, a calorimetric, enzymatic test for cholesterol with lipid clearing factor. HDL cholesterol was measured using human cholesterol liqui-color Phosphatungstic Acid method, end-point kit. Triglycerides were measured using GPO-PAPA $M E T H O D$, a colorimetric, enzymatic method with glycerophosphate oxidase. LDL-cholesterol was computed from the formula: $[\mathrm{LDL}-\mathrm{chol}]=[$ Total chol $]-[\mathrm{HDL}-$ chol] - ([TG]/2.2) where all concentrations are given in $\mathrm{mmol} / \mathrm{L}$. LDL-Cholesterol above $2.0 \mathrm{mmol} / \mathrm{L}$ was considered high.

\section{Urinary albumin}

Creatinine ratio was determined using the CLINITEK Microalbuminuria reagent strips. CLINITEK Microal-2 Strips provided albumin-to-creatinine ratio results in one minute once the strip was placed in an analyzer after being dipped in urine. Estimated glomerular filtration rate was calculated on Cockroft-Gault formula [11].

\section{Data management and statistics}

The data collected were entered into a spreadsheet and cleaned before analysis. Statistical analysis was done in SPSS version 21.0. Descriptive data such as sociodemographic (age, gender, marital status, level of education and employment status) and clinical characteristics (treatment information, Blood Pressure, categories of metabolic control) were summarized into percentages and means/medians. Prevalence of cardiovascular risk factors were presented as proportions of the total number of patients studied or with the risk factor. In addition, variables associated with the cardiovascular risk factors were analyzed. All the associations/comparisons were determined using Chi-square/Fisher's exact test for categorical variables and Student t-test for comparison of means. Odds ratios at $95 \%$ confidence intervals (CIs) were calculated as estimates of relative risks of having poor control of cardiovascular risk factors among patients. Logistic regression model was used to determine independent predictors. Statistical significance was interpreted at a $p$-value of less than 0.05 .

\section{Results}

We enrolled 385 patients with type 2 diabetes into the study. The baseline characteristics are presented in the Tables 1, 2, 3 and 4. The key cardiovascular risk factors were: Hypertension, Obesity, high LDL-cholesterol, Hyperglycaemia and Albuminuria.

There was predominance of female subjects at $65.5 \%$, with mean age 62.1 (12.0) years, younger than the males with 65.7 (11.8) years. The females had a relatively modest formal education, where about $80 \%$ of them had either no education or primary level (less or equal to 7 years in school) compared to $54.9 \%$ of the males with the same.

Almost half of our study population, $49.6 \%$ had hypertension and $69.4 \%$ of them were on either Angiotensin Receptor Blocker (ARBs) or Angiotensin Converting Enzyme Inhibitors (ACEis), 63.0\% on Calcium channel blockers (CCBs) and $47.8 \%$ on a diuretic. Note that there were patients on combinations, CCBs with either ARBs or with ACEis or with diuretics. There were no gender differences.

Regarding treatment of hyperglycaemia, the majority, $68.1 \%$ of the study patients were on oral agents only and $12.0 \%$ were using combined oral agents with insulin. Just $17.1 \%$ were on insulin-only therapy. Glycaemia control was optimal in $39.5 \%$. There were no significant differences in quality of glycaemic control between males and females.

Concerning lipid profile, most patients, $79.2 \%$ had normal HDL, 54.5\% had high triglycerides and $77.1 \%$ had high LDL-cholesterol. Females had higher serum levels of LDL-Cholesterol and total cholesterol than the males. Thirty two (32.7\%) percent had albuminuria.

Using the body mass index, $40 \%$ were overweight (BMI $25.0-29.9 \mathrm{~kg} / \mathrm{m}^{2}$ ) while $22.3 \%$ were obese (BMI $\geq$ $30.0 . \mathrm{kg} / \mathrm{m}^{2}$ ). Other measures of obesity of waist-hip ratio and waist circumference gave different prevalence of obesity, $92.7 \%$ and $58.2 \%$ respectively. The body habitus of high waist circumference meant more abdominal emphasis in most of the study subjects. The physical activity of the study subjects was not quantified in this study.

\section{Discussion}

The burden of type 2 diabetes in sub-Saharan Africa is rising and expected to multiply further, [7] consequently 
Table 1 Clinical and laboratory characteristics of the study subjects

\begin{tabular}{|c|c|}
\hline Variable & Frequency (\%) \\
\hline BMI, mean (SD), kg/m² & $26.7(4.6)$ \\
\hline \multicolumn{2}{|l|}{ Categories, n (\%) } \\
\hline Underweight $(<18.5)$ & $6(1.6)$ \\
\hline Normal (18.5-25) & $139(36.1)$ \\
\hline Overweight (25-29.9) & $154(40.0)$ \\
\hline Obese $(\geq 30)$ & $86(22.3)$ \\
\hline \multicolumn{2}{|l|}{ Blood Pressure (BP) mmHg } \\
\hline $\begin{array}{l}\text { Hypertensive, BP > 140/90 mmHg } \\
\text { or on treatment }\end{array}$ & $191(49.6)$ \\
\hline Normal BP & $194(50.4)$ \\
\hline \multicolumn{2}{|l|}{ Hypertension treatment, Users, } \\
\hline ACEi's/ARBs & $132(69.4)$ \\
\hline Calcium channel blocker(CCBs) & $120(63.0)$ \\
\hline $\begin{array}{l}\text { Diuretics(thiazides, Spironolactone, } \\
\text { Furosemide) }\end{array}$ & $90(47.8)$ \\
\hline \multicolumn{2}{|l|}{ Glucose-lowering treatment } \\
\hline Diet-only & $11(2.9)$ \\
\hline $\begin{array}{l}\text { Oral Glucose-lowering } \\
\text { Agents(OGLAs)-only }\end{array}$ & $262(68.1)$ \\
\hline Insulin-only & $66(17.1)$ \\
\hline $\begin{array}{l}\text { Combined Oral Glucose-lowering } \\
\text { Agents and Insulin }\end{array}$ & $46(12.0)$ \\
\hline \multicolumn{2}{|l|}{ Glycemic control } \\
\hline Mean $\mathrm{HbA1c} \%$ & $8.3(3.0)$ \\
\hline Poor (HbA1c > 7\%) & $233(60.5)$ \\
\hline Good $(\mathrm{HbA} 1 \mathrm{c} \leq 7 \%)$ & $152(39.5)$ \\
\hline Total cholesterol, mean (SD), mmol/L & $4.6(1.2)$ \\
\hline \multicolumn{2}{|l|}{ Categories, n (\%) } \\
\hline High $\geq 4.12$ & $88(22.9)$ \\
\hline Normal, $<4.12$ & $297(77.1)$ \\
\hline $\mathrm{HDL}$, mean (SD), mmol/L & $1.3(0.9)$ \\
\hline \multicolumn{2}{|l|}{ Categories, n (\%) } \\
\hline Low $\leq 1.00$ & $80(20.8)$ \\
\hline Normal $>1.00$ & $305(79.2)$ \\
\hline Triglycerides, mean (SD), mmol/L & $1.7(1.0)$ \\
\hline \multicolumn{2}{|l|}{ Categories, n (\%) } \\
\hline High, $>1.7$ & $210(54.5)$ \\
\hline Normal $\leq 1.7$ & $175(45.5)$ \\
\hline $\mathrm{LDL}$, mean (SD), mmol/L & $2.4(0.9)$ \\
\hline \multicolumn{2}{|l|}{ Categories, n (\%) } \\
\hline High, $>2.0$ & $297(77.1)$ \\
\hline Normal $\leq 2.0$ & $88(22.9)$ \\
\hline \multicolumn{2}{|l|}{ Albuminuria } \\
\hline Albuminuria & $126(32.7)$ \\
\hline Normal & $259(67.3)$ \\
\hline
\end{tabular}

The socio-demographic characteristics of the 385 patients who were included in the study are shown in Table 2 below
Table 2 Socio-Demographic characteristics of the study patients

\begin{tabular}{|c|c|c|c|}
\hline Variable & $\begin{array}{l}\text { Overall } \\
n=385(100 \%)\end{array}$ & $\begin{array}{l}\text { Female } \\
n=252(65.5 \%)\end{array}$ & $\begin{array}{l}\text { Male } \\
n=133(34.5 \%)\end{array}$ \\
\hline Mean age (SD) years & $63.3(12.1)$ & $62.1(12.0)$ & $65.7(11.8)$ \\
\hline \multicolumn{4}{|l|}{ Marital status } \\
\hline Married & $258(67.7)$ & $138(54.8)$ & $120(45.2)$ \\
\hline Separated & $3(0.8)$ & $2(0.8)$ & $1(0.8)$ \\
\hline Single, unmarried & $22(5.7)$ & $19(7.5)$ & $3(2.3)$ \\
\hline Widowed & $102(26.5)$ & $93(36.9)$ & $9(6.8)$ \\
\hline \multicolumn{4}{|c|}{ Level of Formal Education } \\
\hline None & $51(13.2)$ & $48(19.0)$ & $3(2.3)$ \\
\hline $\begin{array}{l}\text { Primary (1-7 years } \\
\text { in school) }\end{array}$ & $227(59.0)$ & $157(62.3)$ & $70(52.6)$ \\
\hline $\begin{array}{l}\text { Secondary } \\
\text { (8-12 years) }\end{array}$ & $90(23.4)$ & $40(15.9)$ & $50(37.6)$ \\
\hline $\begin{array}{l}\text { Tertiary (above } \\
12 \text { years) }\end{array}$ & $17(4.4)$ & $7(2.8)$ & $10(7.5)$ \\
\hline \multicolumn{4}{|l|}{ Employment Status } \\
\hline Employed & 172(44.7) & 123(48.8) & 49(36.8) \\
\hline Unemployed & 213(55.3) & $129(51.2)$ & $84(63.2)$ \\
\hline
\end{tabular}

There was a female preponderance at $65.5 \%$ and more than $70 \%$ of them had either no formal education or a modest one of primary school level

their health sector will face an increasing case-loads of cardiovascular morbidities attributable to diabetes in years to come. The objective of this study was to audit the care of patients with type 2 diabetes attending an out-patient clinic in a public hospital.

There were more females, $65.5 \%$ in our study, probably reflecting gender-related health-seeking behavior in Kenya because prevalence of type 2 diabetes in Kenya,

Table 3 Prevalence of selected cardiovascular risk factors in the study subjects

\begin{tabular}{lll}
\hline Cardiovascular Risk Factors & Prevalence & $95 \% \mathrm{Cl}$ \\
\hline Poor Glycemic control, HbA1c $>7.0 \%$ & $60.5 \%$ & $55.6-65.5$ \\
Poorly controlled Hypertension, & $76.6 \%$ & $72.5-80.8$ \\
$\mathrm{BP} \geq 140 / 90 \mathrm{mmHg}$ & & \\
$\mathrm{LDL}$ Cholesterol, >2.0 mmol/L & $77.1 \%$ & $73.0-81.3$ \\
Obesity, BMI $\geq 30 \mathrm{~kg} / \mathrm{m}^{2}$ & $22.3 \%$ & $18.4-26.5$ \\
Obesity, Waist Circumference & $58.2 \%$ & $53.5-62.9$ \\
$>102 \mathrm{~cm}$ males & & \\
$>88 \mathrm{~cm}$ females & & \\
Obesity, Waist - Hip Ratio & $92.7 \%$ & $89.9-95.3$ \\
$>0.9$ males & & \\
$\quad>0.8$ females & & $27.8-37.4$ \\
Albuminuria & $32.7 \%$ & $19.9-28.5$ \\
Cigarette-smoking, self-reported & $23.6 \%$. &
\end{tabular}

The prevalence of uncontrolled hypertension, high LDL-Cholesterol and obesity (by WHR) was high, above $75 \%$ as shown in the table above 


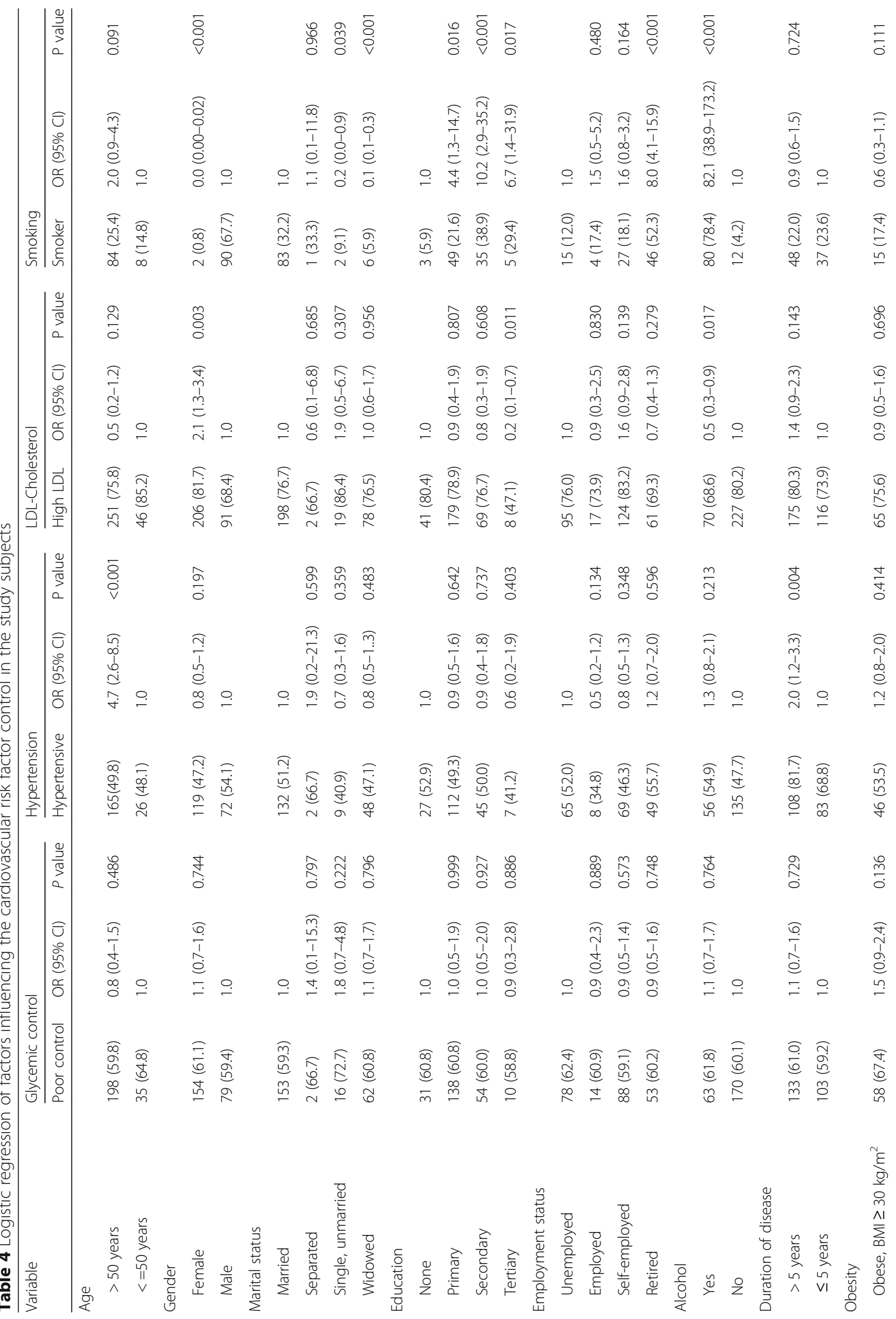




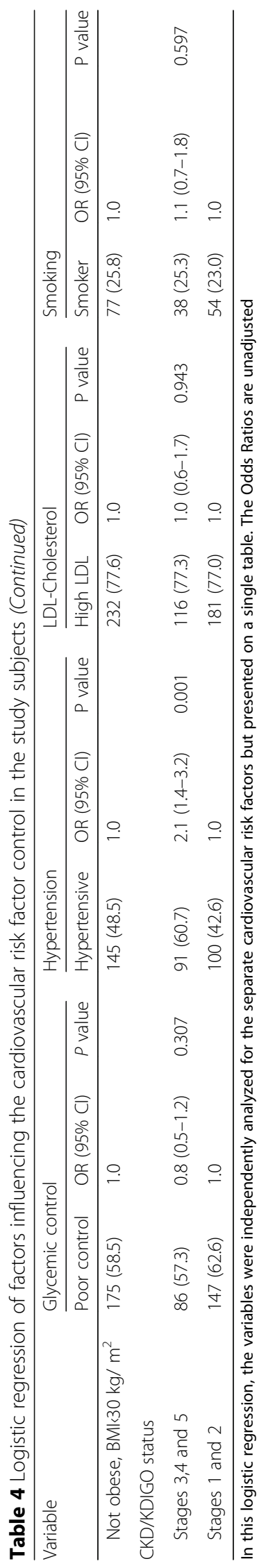


like the study of Ayah R. and Wanjiru R. et al., in Kibera, Nairobi [12], did not show any gender difference in prevalence of diabetes.

Our patients were mainly from rural dwelling, had low formal education where only $27.8 \%$ had attained secondary education and above, in favour of males. The generation of our study population, mean age of 63.3 years, was born in the pre-independent Kenya, when most people did not attend school. Although most of them lacked formal education, they reported self-employment with relative socio-economic stability.

Social determinants of disease in people are important. Socio-economic stability notwithstanding, formal education has a bearing on health literacy. A strong association between low formal education and worse health literacy has been demonstrated in some studies $[13,14]$, which may translate into poor health status [15]. Socio-economic position influences access to care, healthcare behaviour and processes of care [16]. Over $86 \%$ of our patients had visited the clinic 3-4 times in the previous 12 months, meaning they had access to care. It is uncertain how many visits to healthcare provider would be sufficient in a 12-month period in chronic care but the hospital's capacity to offer quality healthcare also counts. Overall, this study registered low proportions of subjects with desired levels of control of: glycaemia of HbA1c $\leq 7 \%$ at $39.5 \%$, Blood pressure $<140$ / $90 \mathrm{mmHg}$ was $23.4 \%$, and Low density lipoprotein cholesterol (LDL-C), $\leq 2.0 \mathrm{mmol} / \mathrm{L}$ was $22.9 \%$. Access to care is better in the County hospitals but the quality of care still falls below expectations. The SMBG is curtailed by frequent changes in glucometer types and their strips from suppliers [17].

The mean HbA1c was $8.2 \%$, with a predominance of poor glycaemic control. The study patients had had diabetes for more than 5 years (mean duration of diabetes of 9.4 years), but only $29 \%$ were on insulin therapy, either in combination or as sole therapy, suggesting that the glucose-lowering treament may not have been intensified. Clinical inertia [18] of care providers and poor adherence to therapies by patients $[19,20]$ are documented contributors to poor glycaemic control amongst patients with diabetes. We found no predictor of glycaemic control amongst the factors analyzed. In our local context, poor adherence is usually occasioned by circumstances of lack of medications in the public hospital and inability of the patients to afford them elsewhere in private pharmacies [17]. Though we did not assess adherence, it was evident that some of our patients were not taking medications prescribed but not available/dispensed in the hospital pharmacy.

Almost half, $49.6 \%$ of the study subjects had hypertension, only $33.4 \%$ of the treated patients were on target at the time of evaluation. The subjects with hypertension were older (64 yrs. versus 58 yrs), had diabetes for longer and a significant proportion at higher stages $(3,4,5)$ of CKD than those without hypertension. The mean systolic and diastolic blood pressures were $143.6 \mathrm{mmHg}$ and $81.4 \mathrm{mmHg}$ respectively. Over two-thirds, $69.0 \%$ were on ACEIs/ARBs, including the subjects that were not on blood pressure targets. It is noteworthy that $66.7 \%$ of patients on ACEi/ARBs had no albuminuria but $33.3 \%$ on similar treatment had albuminuria, OR (95\%CI, 0.7-1.7), $p=0.703$, suggesting no benefit was conferred in mitigating albuminuria. However, we did not ascertain duration of use, doses of and adherence to ACEi/ARB treatment. This was a point evaluation therefore it requires cautious interpretation. Our patients were also using loop-diuretic (Furosemide), $\alpha$ methyldopa and hydralazine because they are relatively cheaper than the desired medications. Gill G., et al. [21] reported that such situations also occur when healthcare providers were not aware of the alternative choices or when the hospital system is not stocking what is appropriate. Hypertension drives kidney disease, it markedly increases the odds of having CKD stages $3-5$, more so in the older age-group, above 50 years as seen in our patients. That hypertension is associated with declining eGFR has been observed, [22-24], is a causative factor of stroke $[25,26]$ and acute coronary syndromes in patients with type 2 diabetes. [27, 28] These emphasize the importance of hypertension control in type 2 diabetes. Framingham study demonstrated that an increment of $20 \mathrm{mmHg}$ in systolic or $10 \mathrm{mmHg}$ in diastolic blood pressure doubled the risk of adverse cardiovascular outcomes across the entire range of blood pressure in people between the ages of 40 to 70 years [29]. Most, $76.6 \%$, of our patients had blood pressures above treatment targets putting them at high risk of cardiovascular events.

Out of $77.1 \%$ with high LDL-C, eligible for treatment, only $12.5 \%$ of our patients were on statin therapy. The females, subjects not on statins and those who were employed had higher serum levels of LDL-cholesterol. The females were also more obese and had higher LDL$C$ levels than the males in this study. Their mean age of 63 years is post-menopausal, and menopause is associated with increased serum LDL-C [30].

High serum LDL-C levels is often asymptomatic, therefore not surprising that low uptake of statins for primary prevention of cardiovascular events is reported $[31,32]$. The reasons advanced to explain low uptake of statins translate to the providers who may not accurately estimate cardiovascular risk, fail to estimate the risk or simply fail to implement guidelines, whose recommendations include periodic assays of serum lipids [32]. We did not determine fidelity to guideline implementation, if there was any in this clinic, but we ascertained that 
lipid assay was not routine in the hospital, attesting to insufficient laboratory support to clinical care.

At the time of evaluation of the five (5) cardiovascular risk factors, namely, poor glycaemic control, hypertension, high LDL-C, cigarette-smoking and obesity, 43.1\% of the subjects had any two (2) risk factors under optimal control. Only $20.3 \%$ subjects had the three (3) main risk factors of hyperglycaemia, hypertension and high LDL-C simultaneously in optimal control at the time of this study, which was rather dismal. The care context observed during the study was simplistic in organization, insufficient in staff empowerment, had sub-optimal quality of diabetes self-management education and inadequate laboratory support to care therefore not supportive of multiple risk factor reduction. Beran D., et al. [33] and Whiting D., et al. [34] in studies also in sub-Saharan Africa, made similar observations.

Evidence has shown that intensified cardiovascular risk factor control in patients with type 2 diabetes, either as single or double risk factor reduction, significantly mitigates morbidity and mortality [35-40]. However, Drake et al. [41], re-analyzing the ACCORD study, demonstrated that over $30 \%$ of the study subjects who had not achieved treatment targets were due to clinical and demographic reasons that highlighted the inherent challenges underlying the care of diabetes. Regarding our patients, it is also probable, amongst other reasons that we did not study, that treatment intensification and risk stratification of patients were not done.

Reducing multiple cardiovascular risk factors simultaneously in subjects with type 2 diabetes has already been demonstrated as a feasible strategy [42-45], but with overwhelming support to systems of care during the clinical trials. Those levels of gain have not been replicated in routine care. For instance, lowering SBP by $4 \mathrm{mmHg}$, LDL-C by $1 \mathrm{mmol} / \mathrm{L}$ and HbAlc by $0.9 \%$ reduced cardiovascular events by $12.5 \%, 8.2 \%$ and $2.9 \%$ respectively [46-48], additional to other good lifestyle practices over time. Wong $\mathrm{N}$, et al., further showed that reducing these risk factors simultaneously in the patients with type 2 diabetes incrementally lowered cardiovascular event rates [49]. Those three main risk factors drive cardiovascular events and their control confers relative cost-benefits on diabetes-associated outcomes. Self-care in patients with diabetes should be sufficient to address the multiple risk factors that one may have. However, this is often a pipedream. Studies in sub-Saharan Africa, like Ethiopia [50, 51] and Nigeria [52] reported poor self-care behaviors, including poor adherence to therapies. One quarter of our patients were cigarette-smokers, mainly men who under-reported the habit. Cigarettesmoking is an important cardiovascular risk whose remedy is reported to be challenging in Africa [53, 54]. Sub-Saharan Africa is not achieving goals of control of non-communicable diseases [55]. From this study, it is probable that social determinants may be playing a big role, especially the low formal education, high unemployment and consequent low economic capacity.

In the sub-Saharan Africa, the challenges of diabetes care occur at both individual level and healthcare systems that favor acute care over chronic care. The national policies are skewed and capacities in disposable resources attenuated. These factors conspire to deter achievement of optimal control of the different cardiovascular risk factors simultaneously [56]. It is imperative that care strategies that achieve simultaneous control of cardiovascular risk factors to targets may be the main challenge or probably the missing link between the clinical care we offer and desired gains we should receive. Compared to instruments of care and new agents, delivery of care may be the bigger problem in sub-Saharan Africa. We recommend innovative designs of health systems and care delivery which would comprise reliable financing, functional internal and external system linkages, efficient teams with appropriate care attitudes and stewardship. These should form the next frontiers of operations research to inform clinical management of patients with type 2 diabetes who access care.

\section{Conclusion}

We found high prevalence of cardiovascular risk factors in our patients who attended the public hospital clinic and they were inadequately controlled. The reasons settle down to sub-optimal quality of care because of modest socio-economic status of most patients, insufficient resources to support clinical staff and laboratory to meet care requirements. This put a large proportion of the patients at risk of cardiovascular events with potentially obvious challenges in their care. This is a common picture in many public health facilities that the majority of our patients visit for both acute and chronic care.

\section{Abbreviations \\ CKD/KDIGO: Chronic Kidney disease/Kidney Disease Improving Global \\ Outcomes; IQR: Interquartile range}

\section{Acknowledgements}

We acknowledge and thank all the Nursing and Medical staff at the Nyeri County hospital for facilitating the study. We are truly grateful to the patients who took time to participate in the survey. Last, but not least, we give gratitude to Dorcas, Secretary of the Department of Clinical Medicine and Therapeutics for typing and formatting the manuscript.

\section{Funding}

The study was sponsored by the investigators, CFO and MWK, from personal resources. No funding agency was involved in the study.

Availability of data and materials

The tabulated raw data is available and uploaded.

Authors' contributions

FCFO, ENO, MWK and KM conceptualized the study. MWK collected the data. FCFO made the initial drafts of the manuscript and KM did the statistics, ENO 
made critical revision to the manuscript but all authors contributed to the final manuscript writing. All authors read and approved the final manuscript.

\section{Ethics approval and consent to participate}

This study was approved for conduct by the University of Nairobi/Kenyatta National Hospital Ethical Review Board (ERB), (the body nationally mandated to approve clinical studies) after it was peer-reviewed by the academic staff of the Department of Medicine. The administration of the Nyeri County hospital also approved the study and allowed sharing of the results with clinical staff for patient care. The participating patients gave written consent.

\section{Consent for publication}

The Ethics and Review Board of University of Nairobi/Kenyatta National Hospital gave the approval to publish the study findings.

\section{Competing interests}

The authors declare that they have no competing interests.

\section{Publisher's Note}

Springer Nature remains neutral with regard to jurisdictional claims in published maps and institutional affiliations.

\section{Author details}

${ }^{1}$ Department of Clinical Medicine and Therapeutics, College of Health Sciences, University of Nairobi, Nairobi, Kenya. ${ }^{2}$ Kenyatta National Hospital, Nairobi, Kenya.

Received: 29 March 2017 Accepted: 20 November 2017 Published online: 01 December 2017

\section{References}

1. Kuller LH, Velentgas P, Barzilay J, et al. Diabetes mellitus: sub-clinical cardiovascular disease and risk of incident cardiovascular disease and allcause mortality. Arterioscler Thromb Vasc Biol. 2000:20:823-9.

2. Almdal T, Scharling $H$, Jensen JS, Vestergaard $H$. The independent effect of type 2 diabetes mellitus on ischemic heart disease, stroke, and death: a population-based study of 13000 men and women with 20 years of followup. Arch Intern Med. 2004;164:1422-6.

3. Casagrande SS, Fradkin JE, Saydah SH, Rust KF, Cowie CC. The prevalence of meeting A1c, blood pressure and LDL goals among people with diabetes, 1988-2010. Diabetes Care. 2013;36:2271-9.

4. International Diabetes Federation. The IDF diabetes atlas. 6th edn. Brussels: International Diabetes Federation, 2013. http://www.idf.org/sites/default/ files/EN_6E_Atlas_FULL_0.pdf (accessed on 10 Oct 2016).

5. Yusuf S, Hawken S, Ounpuu S, Dans T, Avezum A, Lanas F, et al. Effect of potentially modifiable risk factors associated with myocardial infarction in 52 countries (the INTERHEART study): case-control study. Lancet. 2004;364:937-52.

6. Adeniyi OV, Yogeswaran P, Longo-Mbenza B, Goon DT, Ajayi Al, et al. Crosssectional study of patients with type 2 diabetes in OR Tambo district, South Africa. BMJ Open. 2016;6:1-8.

7. Paul A, Oparil S, Carter B. Report from the panel members appointed to the eighth joint National Committee (JNC 8). JAMA. 2014;311(5):507-20.

8. Waist circumference and waist-hip ratio: report of a WHO expert consultation, Geneva, 8-11 December 2008. Chapter 2: Methods for measuring waist and hip circumference, page 5-7. (c) World Health Organization 2011

9. Expert Panel on Detection, Evaluation, and Treatment of High Blood Cholesterol in Adults. Executive summary of the third report of the National Cholesterol Education Program (NCEP) expert panel on detection, evaluation, and treatment of high blood cholesterol in adults (adult treatment panel III). JAMA. 2001;285:2486-97.

10. World Health Organisation. Obesity: preventing and managing the global epidemic: report of a WHO consultation. Geneva: WHO Technical Report Series 894; 2000

11. Rostoker G, Andrivet P, Pham I, Griuncelli M, Adnot S. A modified Cockcroft Gault formula taking into account the body surface area gives a more accurate estimation of the glomerular filtration rate. J Nephrol. 2007;20(5):576-85.

12. Ayah R, Wanjiru R, Otieno CF, Joshi MD, et al. A population-based survey of prevalence of diabetes and correlates in an urban slum community in Nairobi, Kenya. BMC Public Health. 2013;13:371.
13. Baker DW, Peter RM, Williams MV, et al. Health literacy and the risk of hospital admissions. J Gen Intern Med. 1998;13:791-8.

14. Schillinger D, Grumbach K, Piette J, et al. Associations of literacy with diabetes outcomes. JAMA. 2002;288:475-82.

15. Street RL Jr, Piziak VK, Carpentier WS, et al. Provider-patient communication and metabolic control. Diabetes Care. 1993;16:714-21.

16. Brown AF, Ettner SL, Piette J, Weinberger M, Gregg E, Shapiro MF, Kartner AJ, et al. Socioeconomic position and health among persons with diabetes mellitus: a conceptual framework and review of literature. Epidemiologic Rev. 2004;26:63-77.

17. Mwavua SM, Ndungu EK, Mutai KK, Joshi MD. A comparative study of the quality of care and glycaemic control among ambulatory type 2 diabetes clients at a tertiary referral hospital and a regional hospital in Central Kenya. BMC Res Notes. 2016;9:12. doi:10.1186/s13104-015-1826-0.

18. Grant RW, Cagliero E, Dubey AK, et al. Clinical inertia in the management of type 2 diabetes metabolic risk factors. Diabetic Med. 2004;21(2):150-5.

19. Rubin RR, et al. Adherence to pharmacologic therapy in patients with type 2 diabetes mellitus. Am J of Med. 2005;118(Suppl5A):275-345.

20. Bailey CJ, Kodack M. Patient adherence to medication requirements for therapy of type 2 diabetes. Int J Clin Pract. 2011;65(3):314-22.

21. Gill G. Diabetes in Africa - puzzles and challenges. Indian J Endocrinol Metabol. 2014;18(3):249-51.

22. Ratnekaran R, Cull CA, Thorne Kl, Adler Al, Holman RR. Risk factors for renal dysfunction in type 2 diabetes: UKPDS 74. Diabetes. 2006;55:1832-8.

23. Zoppini G, Targher G, Chonchol M, Ortalda V, Negri C, Stoico V, et al. Predictors of estimated Glomerular filtration rate in patients with type 2 diabetes and preserved kidney function. Clin J Am Soc Nephrol. 2012;7:401-8.

24. Takagi M, Babazono T, Uchigata Y. Differences in risk factors for the onset of albuminuria and decrease in GFR in people with type 2 diabetes: implications for the pathogenesis of diabetic kidney disease. Diabetic Med. 2015:32:1352-60.

25. Davis TM, Millens H, Stratten JM, et al. Risk factors for stroke in type 2 diabetes mellitus: United Kingdom prospective diabetes study (UKPDS) 29 Arch Intern Med. 1999:159:1097-103.

26. Straus SE, Majumdar SR, McAlister FA. New evidence for stroke prevention. JAMA. 2002;288:1388-95.

27. Lee MG, Jeong MH, Lee HK, Park HK, Sim DS, et al. Prognostic impact of diabetes mellitus and hypertension for mid-term outcome of patients with AMI who underwent PCI. Cardiology. 2012;60(4):257-63.

28. Nguchu HK, Joshi MD, Otieno CF. Acute coronary syndromes amongst type 2 diabetics with ischaemic electrocardiograms presenting to accident and emergency Department of a Kenyan tertiary institution. East Afr Med J. 2009:86(10):463-8.

29. Christopher J, Elosua R. Cardiovascular risk factors. Insights from Framingham heart study. Rev Esp Cardiol. 2008;61(3):299-310.

30. Matthews KA, Meilahn E, Kuller LH, Kelsehy SF, Caggiula AW, Wing RR. Menopause and risk factors for coronary heart disease. N Engl J Med. 1989;321:641-6.

31. Otieno CF, Vaghela V, Mwendwa F, Amayo EO, Ogola EN. Cardiovascular risk factors in patients with type 2 diabetes mellitus in Kenya: levels of control attained at the outpatient diabetic clinic of Kenyatta National Hospital, Nairobi. East Afr Med J. 2005:82(12):S184-90.

32. Berthold HK, Gouni-Berthold I, Bohm M, Krone W. Patterns and predictors of statin prescription in patients with type 2 diabetes. Cardio Diabetol. 2009:8:25.

33. Beran D, Yudkin JS. Diabetes care in sub-Saharan Africa. Lancet. 2006;368: 1689-95.

34. Whiting D, Hayes $L$, Unwin N. Diabetes in Africa. Challenges to care for diabetes in Africa. J Cardiovasc Risk. 2003;10:103-10.

35. UKPDS Group. Intensive blood glucose control with sulfonylurea or insulin compared with conventional treatment and risk of complications in patients with type 2 diabetes. UKPDS 33. Lancet. 1998;352(9131):837-53.

36. The ADVANCE Collaborative Group. Intensive blood glucose control and vascular outcomes in patients with type 2 diabetes. New Engl J Med. 2008;358(24):2560-972.

37. ALLHAT Collaborative Research Group. Major outcomes in high-risk hypertensive patients randomized to ACEis or calcium channel blockers versus diuretic therapy. The ALLHAT study. JAMA. 2002;288(23):2981-97.

38. Collins R. Heart protection study collaborative group: MRC/BHF heart protection study of cholesterol-lowering with simvastatin in 20,536 high-risk individuals: a randomized placebo controlled trial. Lancet. 2002:360:7-22.

39. ACCORD Study Group, Cushman WC, Evans GW, Byington RP, et al. Effects of intensive blood-pressure control in type 2 diabetes mellitus. $N$ Engl J Med. 2010;362:1575-85. 
40. Margolis KL, O'Connor PJ, Morgan TM, Buse JB, et al. Outcomes of combined cardiovascular risk management strategies in type 2 diabetes mellitus. Diab Care. 2014;37:1721-8.

41. Drake TC, Hsu FC, Hire D, Chen S-H, Cohen RM, McDuffie R, Nylen E, O'Connor $P$, Rehman S, Seaquist RS. Factors associated with failure to achieve a glycated haemoglobin target of $<8.0 \%$ in the action to control cardiovascular risk in diabetes (ACCORD) trial. Diabetes Obes Metab. 2016;18:92-5.

42. Gaede $\mathrm{P}$, Lund-Andersen $\mathrm{H}$, Parving $\mathrm{HH}$, Pederson $\mathrm{O}$. Effect of multi-factorial intervention on mortality in type 2 diabetes. N Engl J Med. 2008;358:580-91.

43. Gaede P, Oellgaard J, Carstensen B, Lund-Andersen H, Parving HH, Pederson O. Years of life gained by multifactorial intervention in patients with type 2 diabetes and microalbuminuria: 21 years follow-up on Steno-2 randomised trial. Diabetologia 2016; MID:27531506. DOl:10.1007/s00125-016-4065-6.

44. Bittner V, Bertolet $M$, Barraza Felix R, et al. Comprehensive cardiovascular risk factor control improves survival - the BARI-2D trial. J Am Coll Cardiol. 2015;66(7):765-73

45. Moreno-Palanco MA, Ibanez-Sanz P, Pablo CC, Pizzaro-Portillo A, RodriguezSalvanes F, Suarez-Fernandez C. Impact of comprehensive and intensive treatment of risk factors concerning cardiovascular mortality in secondary prevention: MIRVAS study. Rev Esp Cardiol. 2011;64(3):179-85.

46. McLarren JM, Sattar N. The importance of a comprehensive multifactorial treatment approach in type 2 diabetes. Medicographia. 2016;38(1):20-7.

47. Ray KK, Seshasai SRK, Wijesuriya S, Sivakumaran R, Nethercott S, et al. Effect of intensive control ofglucose on cardiovascular outcomes and death in patients with diabetes mellitus: a meta-analysis of randomised controlled trials. Lancet. 2009:373(9677):1765-72.

48. Ferguson LD, Sattar N. Reducing cardiovascular disease risk in type 2 diabetes: is the focus on glycaemia warranted? Diabetes Obes Metabol. 2013;15(5):387-91.

49. Wong ND, Zhao Y, Patel R, Patao C, Malik S, Bertoni AG, Correa A, Folsom AR, Kachroo S, Mukherjee J, Taylor H, Selvin E. Cardiovascular risk factor targets and cardiovascular disease event risk in diabetes: a pooling project of the atherosclerosis risk in communities study, multi-ethnic study of atherosclerosis, and Jackson heart study. Diabetes Care. 2016;39(5):668-76.

50. Kassahun T, Gesesew K, Mwanri L, Eshetie T. Diabetes-related knowledge, self-care behaviours and adherence to medications among diabetic patients in Southwest Ethiopia. A cross-sectional survey. BMC Endocr Disord. 2016;16: 28. doi:10.1186/s12902-016-0114-x.

51. Tewahido D, Berhane $Y$. Self-care practices among diabetes patients in Addis Ababa. A qualitative study. PLoS One. 2017;12(1):e0169062. doi:10.1371/journal.pone.0169062.

52. Okafor Cl, Ofoegbu EN. Control of cardio-metabolic risk factors among Nigerians living with type 2 diabetes mellitus. Nigerian J Clin Practice. 2012;15(1):15-8.

53. Sreeramareddy CT, Pradhan PM, Sin S. Prevalence, distribution, and social determinants of tobacco use in 30 sub-Saharan African countries. BMC Med. 2014;12:243. doi:10.1186/s12916-014-0243-X.

54. Hosseinpoor AR, Parker LA, D'Espaignet ET, Chatterii S. Socio-economic inequality in smoking in low-income and middle-income countries: results from the world health survey. PLoS One. 2012;7:e42843.

55. Nyaaba GN, Stronks K, de Graft Aikins A, Kengne AP, Agyemang C. Tracing Africa's progress towards implementing the non-communicable diseases global action plan 2013-2020: a synthesis of WHO country profile reports. BMC Public Health. 2017;17:297. doi:10.1186/s12889-017-4199-6.

56. BeLue R, Okoror TA, Iwelunmor J, Taylor KD, Degboe AN, et al. An overview of cardiovascular risk factor burden in sub-Saharan African countries. A sociocultural perspective. Glob Health. 2009:5:10. doi:10.1186/1744-8603-5-10.

\section{Submit your next manuscript to BioMed Central and we will help you at every step:}

- We accept pre-submission inquiries

- Our selector tool helps you to find the most relevant journal

- We provide round the clock customer support

- Convenient online submission

- Thorough peer review

- Inclusion in PubMed and all major indexing services

- Maximum visibility for your research

Submit your manuscript at www.biomedcentral.com/submit
Biomed Central 